\title{
The role of PET in a clinically silent and ultrasound negative synovitis in the course of rheumatoid arthritis - a case report
}

\author{
M. Skoczynska ${ }^{1,2}$, F. Figus ${ }^{1}$, V. Arena ${ }^{3}$, G. Massazza ${ }^{4}$, A. lagnocco ${ }^{1}$ \\ ${ }^{1}$ Academic Rheumatology Centre and Dipartimento Scienze Cliniche e Biologiche, \\ Università degli Studi di Torino, Turin, Italy; ${ }^{2}$ Department and Clinic of Rheumatology and Internal Diseases, \\ Wroclaw Medical University, Wroclaw, Poland; ${ }^{3}$ Affidea Irmet Torino, Turin, Italy; \\ ${ }^{4}$ Division of Physical Medicine and Rehabilitation, MFRU, Università degli Studi di Torino, Turin, Italy
}

\section{SUMMARY}

We present a case report of a rheumatoid arthritis patient, who underwent a PET scan, which revealed inflammation of multiple joints, which was missed by both physical and ultrasound examinations.

A 55-year old woman with a long-term rheumatoid arthritis, who had undergone arthroplasty of the left knee in the past, consulted with the rheumatologist for pain in the left knee. The physical examination revealed signs of inflammation in the left knee and right elbow. The inflammatory parameters were high. Ultrasound showed intraarticular effusion without signs of active synovitis in the left knee. The ultrasound assessment of the other joints (hands, wrists and feet) was also negative for active synovitis, while positron-emission tomography (PET) revealed increased glucose metabolism at the level of the medial side of the left knee, left radio-ulno-carpal joint, I-II-III metacarpo-phalangeal joints bilaterally, right II metatarso-phalangeal joint, and left II-III metatarso-phalangeal joints.

This case report demonstrates that PET might be more sensitive than ultrasound in detecting subclinical joint inflammation.

Key words: Rheumatoid arthritis; positron-emission tomography; ultrasonography.

Reumatismo, 2021; 73 (1): 64-66

\section{INTRODUCTION}

The established 'gold standard' in rheumatoid arthritis (RA) disease activity evaluation includes clinical assessment and ultrasound and/or magnetic resonance imaging, as recommended by the European Alliance of Associations for Rheumatology (EULAR) (1). Although metabolic changes shown by positron-emission tomography (PET) are not specific for a particular type of joint inflammation, they have been reported to correlate well with the clinically- and sonographically-assessed disease activity $(2,3)$.

Corresponding author:

Annamaria lagnocco

Academic Rheumatology Centre, MFRU and Dipartimento

Scienze Cliniche e Biologiche Università degli Studi di Torino, Turin, Italy E-mail: annamaria.iagnocco1@gmail.com

\section{CASE REPORT}

We present the case of a patient with clinically asymptomatic joints and negative ultrasound who was found to be PET-positive for joint inflammation. A 55 year old wom- an, diagnosed with seropositive RA (2006) and osteoarthritis, who had undergone arthroplasty of the left knee in 2011, presented to the rheumatologist in the summer of 2018 due to pain in the left knee occurring predominantly during the day, accompanied by morning stiffness lasting about 30 minutes and reduced range of movement. The patient's global assessment on a 0-10 $\mathrm{cm}$ visual analogue scale (VAS) was $8 \mathrm{~cm}$. Recent blood tests showed increased inflammatory parameters: ESR $38 \mathrm{~mm} / \mathrm{h}$ (nv $<20 \mathrm{~mm} / \mathrm{h}$ ), CRP $47.8 \mathrm{mg} / \mathrm{dl}(\mathrm{nv}<5 \mathrm{mg} /$ dL), WBC 14 500/L (nv <10 000/L), NEU 11 480/L (nv <8000/L). Physical examination revealed signs of joint inflammation in the left knee and right elbow. The disease activity according to disease activity score 28 (DAS28) was 5.03.

The left knee X-ray showed a lateralization of the prosthetic tibial component with periprosthetic bone reabsorption. Ultra- 
sound performed using a General Electric Logiq E9 machine, equipped with a 9-15 $\mathrm{MHz}$ broadband linear array transducer, showed intra-articular effusion without signs of active synovitis in the left knee. Ultrasound of the other joints (hands, wrists and feet) was also negative for active synovitis. Since a left knee septic arthritis was suspected, the patient was referred for fluorine-18 fluorodeoxyglucose PET, which revealed scarse tracer accumulation at the medial side of the left knee (not in the prosthetic area) and an evident accumulation at the level of left radio-ulno-carpal joint, I-II-III metacarpo-phalangeal joints bilaterally, right II metatarso-phalangeal joint, and left II-III metatarso-phalangeal joints (Figure 1).

\section{DISCUSSION}

Due to the scarcity of published data on the use of PET in RA, this technique was not mentioned in the 2013 EULAR Recommendations for the use of imaging in the clinical management of RA (1). However, over the last few years, new studies were published that suggest a potentially important role for PET in RA patient assessment. In 2014, dos Anjos and da Mota published a systematic literature review on this subject, describing the results of fifteen articles (twelve original studies and three case reports), which show that PET correlates well with the disease activity indexes in patients with a defined RA diagnosis (4). However, it is yet to be established whether PET may be useful in the differentiatial diagnosis of RA from non-RA pathologies. In one study, quantitative parameters such as maximum standardized uptake value, metabolic active volume, and total lesion glycolysis, as well as total visual score calculated based on those parameters, were significantly different in RA patients compared to patients with other types of artritis (5).

PET seems to be a highly sensitive method to assess disease activity both in early RA and at follow-up $(2,3)$. In one study, PET findings related to the presence of joint activity were in agreement with both the ultrasound and MRI evidence of inflam-

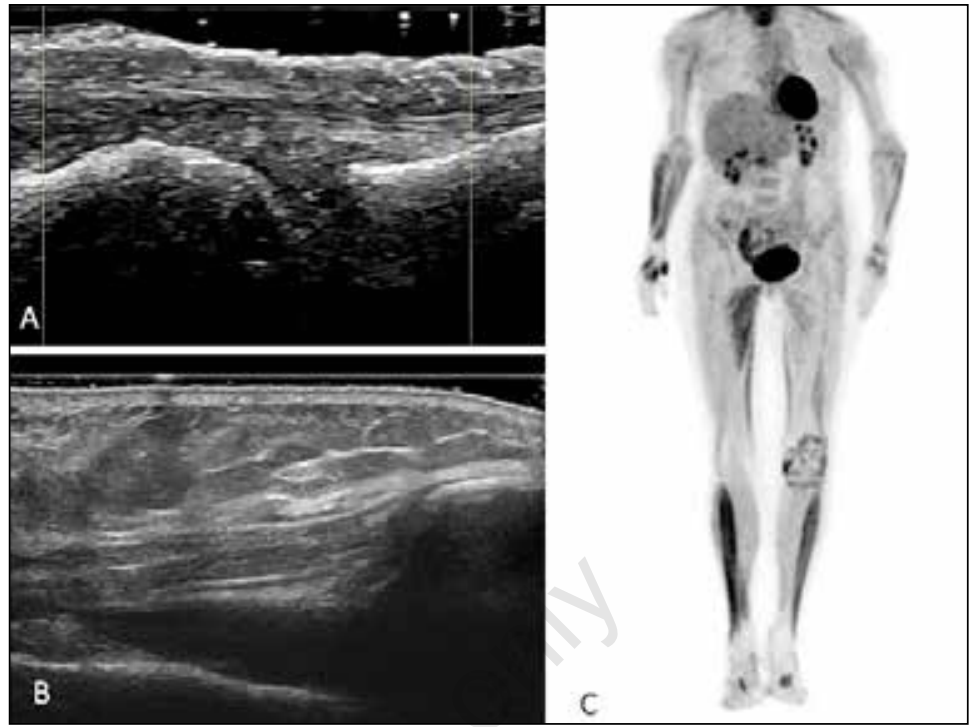

Figure 1 - Ultrasound and PET images of the patient. A. Right hand II metacarpo-phalangeal joint with no signs of active synovitis. B. Left knee suprapatellar compartment with effusion, but no signs of active synovitis. C. Fluorine-18 fluorodeoxyglucose PET scan revealing increased metabolic activity at the level of left radio-ulno-carpal joint, I-II-III metacarpo-phalangeal joints bilaterally, medial side of the left knee, right II metatarso-phalangeal joint and left II-III metatarso-phalangeal joints.

mation, as well as with laboratory findings such as CRP and serum MMP-3 levels (3). In another study, the number of PET-positive joints was found to correlate with the total number of tender and swollen joints. The authors developed a validated PET/ DAS score, calculated as $(0.063 \times$ number of PET-positive joints in 28 joints $)+(0.011$ $\times$ ESR $)+(0.030 \times$ PtGA) (patient's global assessment of disease activity), which correlated with DAS28-ESR ( $\mathrm{p}<0.001)$. PETpositive joints were defined as joints with FDG uptake greater than normal regional tracer accumulation in areas corresponding to the joint synovium. The authors argued that the PET/DAS score could be used as a more objective tool than the clinical evaluation for disease activity assessment, especially in clinical trials and research studies (2).

Aside from its potential diagnostic value, PET seems to have a promising role as a predictive tool in RA patients. In subclinically inflamed joints, it has been demonstrated that macrophage targeting PET using (R)-11C-PK11195 tracer may predict 
arthritic flares. In a study from Gent et al. performed on early RA patients with subclinical synovitis, higher cumulative PET scores were associated with subsequent flare in hand or wrist, which on the contrary did not correlate with MRI scores (6). Furthermore, PET results were shown to correlate with the progression of radiographic changes (7) and the response to both conventional and biologic disease modyfying drugs $(2,4)$. PET could be included in the stratification of RA flare risk and therapy personalization so as to initiate earlier a more aggressive treatment, possibly with biologic agents, in patients identified as being at a high risk of flare or radiographic progression, and earlier DMARD and glucocorticoid dose tapering in patients at lower risk of recurrent arthritis. Lastly, in RA patients, PET shows active inflammation in both articular and extra-articular sites, including regional lymph nodes, shedding some light on RA pathophysiology.

The use of PET in RA evaluation is potentially limited by relatively high costs and low accessibility. In addition, higher tracer uptake is not limited to sites of inflammation. Another limitation of PET with fluorine-18 fluorodeoxyglucose, a marker of glucose metabolism, is that false positive results may be obtained after physical activity or conditions associated with increased muscle tension due to higher muscle metabolic activity as well as false positive readings for adjacent joints.

Although the routine use of PET in the clinical pratice may have some limitations, this case report demonstrated that PET is superior to ultrasound in the detection of synovitis. More studies are needed to establish the future role of PET in the management of patients with RA and other rheumatic diseases, however its use in selected cases can be valuable.

\section{Authors' contributions}

MS revised the literature and wrote the draft of the manuscript; FF evaluated the clinical case and wrote the revised version of the manuscript; VA provided PET images and critically reviewed the manuscript; GM critically reviewed the manuscript; AI designed the idea of the manuscript, provided ultrasound images and critically reviewed the manuscript. All authors declare no conflict of interests.

\section{Informed consent}

Patient's informed consent to publish this material was obtained.

\section{REFERENCES}

1. Colebatch AN, Edwards CJ, Østergaard M, et al. EULAR recommendations for the use of imaging of the joints in the clinical management of rheumatoid arthritis. Ann Rheum Dis. 2013; 72: 804-14.

2. Lee SJ, Jeong JH, Lee CH, et al. Development and validation of an 18 F-FDG PET/CT-based tool for the evaluation of joint counts and disease activity in patients with rheumatoid arthritis. Arthritis Rheumatol. 2019; 71: 123240.

3. Beckers C, Jeukens X, Ribbens C, et al. (18)FFDG PET imaging of rheumatoid knee synovitis correlates with dynamic magnetic resonance and sonographic assessments as well as with the serum level of metalloproteinase- 3 . Eur J Nucl Med Mol Imaging. 2006; 33: 27580.

4. dos Anjos DA, da Mota LM. Positron emission tomography with (18)F-FDG in the evaluation of patients with rheumatoid arthritis - a systematic review (in Portuguese). Rev Bras Reumatol. 2014; 54: 474-82.

5. Bhattarai A, Nakajima T, Sapkota S, et al. Diagnostic value of $18 \mathrm{~F}$-fluorodeoxyglucose uptake parameters to differentiate rheumatoid arthritis from other types of arthritis. Medicine (Baltimore) 2017; 96: e7130.

6. Gent YY, Ter Wee MM, Voskuyl AE, et al. Subclinical synovitis detected by macrophage PET, but not MRI, is related to short-term flare of clinical disease activity in early RA patients: an exploratory study. Arthritis Res Ther. 2015; 17: 266.

7. Suto T, Okamura K, Yonemoto Y, et al. Prediction of large joint destruction in patients with rheumatoid arthritis using 18F-FDG PET/CT and disease activity score. Medicine (Baltimore). 2016; 95: e2841. 